\title{
Defining replication origin efficiency using DNA fiber assays
}

\author{
Sandie Tuduri • Hélène Tourrière $\cdot$ Philippe Pasero
}

Published online: 29 December 2009

(C) Springer Science+Business Media B.V. 2009

\begin{abstract}
The timely duplication of eukaryotic genomes depends on the coordinated activation of thousands of replication origins distributed along the chromosomes. Origin activation follows a temporal program that is imposed by the chromosomal context and is under the control of S-phase checkpoints. Although the general mechanisms regulating DNA replication are now well-understood at the level of individual origins, little is known on the coordination of thousands of initiation events at a genome-wide level. Recent studies using DNA combing and other single-molecule assays have shown that eukaryotic genomes contain a large excess of replication origins. Most of these origins remain "dormant" in normal growth conditions but are activated when fork progression is impeded. In this review, we discuss how DNA fiber technologies have changed our view of eukaryotic replication programs and how origin redundancy contributes to the maintenance of genome integrity in eukaryotic cells.
\end{abstract}

Keywords DNA combing - replication forks · initiation $\cdot$ checkpoints

Responsible Editor: Marie-Noëlle Prioleau and Dean Jackson.

S. Tuduri $\cdot H$. Tourrière $\cdot P$. Pasero $(\bowtie)$

Institute of Human Genetics, CNRS UPR 1142,

141 rue de la Cardonille,

34396 Montpellier Cedex 5, France

e-mail: ppasero@igh.cnrs.fr

\author{
Abbreviations \\ ARS Autonomously replicating sequence \\ BrdU Bromo-deoxyuridine \\ CldU Chloro-deoxyuridine \\ IdU Iodo-deoxyuridine \\ EM Electron microscopy \\ FISH Fluorescent in situ hybridization \\ HU Hydroxyurea \\ IOD Inter-origin distance \\ ORC Origin recognition complex \\ Pre-RC Pre-replication complex \\ rDNA Ribosomal DNA
}

\section{Introduction}

Eukaryotic DNA replication is initiated from specific sites called replication origins, which are scattered along the chromosomes. This process must be tightly regulated to ensure that the entire genome is entirely replicated once and only once per cell cycle. Initiation of DNA replication is controlled through a two-step process that has been conserved throughout evolution. A pre-replication complex (pre-RC) is first assembled on replication origins during the $G_{1}$ phase of the cell cycle and is subsequently activated during $\mathrm{S}$ phase by specific protein kinases (Bell and Dutta 2002). Pre$\mathrm{RC}$ assembly consists in the loading of the Mcm2-7 hexamer at replication origins in an origin recognition complex (ORC)-, Cdc6-, and Cdt1-dependent manner. 
Origin firing in $\mathrm{S}$ phase depends on the coordinated action of cyclin-dependent kinases (CDKs) and Dbf4dependent kinases (DDKs). Phosphorylation of a limited number of initiation factors by CDKs and DDKs triggers the unwinding of the DNA duplex at origins and allows the engagement of the replication machinery (Tanaka et al. 2006; Zegerman and Diffley 2006). Importantly, high CDK activity also blocks pre-RC assembly and prevents reinitiation until the next cell cycle (Arias and Walter 2007). Upon initiation, the Mcm2-7 complex acts as an ATPdependent DNA helicase to promote the bidirectional progression of replication forks, together with $\mathrm{Cdc} 45$, GINS, and other components of the replisome (Gambus et al. 2006). Replisomes progress along parental DNA until they encounter converging forks.

Although considerable progress has been made in understanding the cell cycle regulation of replication initiation, the mechanisms that coordinate the sequential firing of thousands of replication origins throughout $\mathrm{S}$ phase remain unclear. In all the eukaryotes, origin activation follows a temporal program that is stably transmitted to daughter cells (Hiratani et al. 2009; Lucas and Feng 2003). Evidence from budding yeast indicates that replication timing is not determined genetically but is imposed by the chromosomal environment of origins. Hence, early origins replicate late when they are moved to late-replicating regions of the genome, whereas initiation at late origins is advanced when they are moved to early regions (Lucas and Feng 2003). Multiple factors affect replication timing in eukaryotic cells, including histone modifications, subnuclear localization, and gene activity (Hiratani et al. 2009; Knott et al. 2009; Lucas and Feng 2003). Replication timing is also controlled by checkpoint kinases, which detect stalled replication forks and prevent the activation of late origins until the replication stress is relieved (Tourriere and Pasero 2007).

Replication forks frequently stall during a normal S phase. Fork arrest is caused by multiple events, such as DNA lesions, tightly bound protein complexes, and transcription at highly expressed genes (Azvolinsky et al. 2009; Tourriere and Pasero 2007; Tuduri et al. 2009). Cells have evolved efficient fork repair mechanisms to restart stalled forks. These mechanisms are particularly active in prokaryotes, which rely on a single pair of replication forks to duplicate their genome (Michel et al. 2007). In contrast, eukaryotes have adopted a different strategy to deal with replication stress, which is based on origin redundancy rather than fork restart at any cost. This is best illustrated with the Saccharomyces cerevisiae genome, which is only three times larger than the one of Escherichia coli but contains more than 300 replication origins (Raghuraman et al. 2001). Replication origins are also present in large excess in higher eukaryotes. Most of these so-called dormant origins are not used in normal growth conditions but fire when DNA replication is challenged with genotoxic drugs (Ge et al. 2007; Ibarra et al. 2008). Origin redundancy appears therefore an important mechanism preventing genomic instability during $\mathrm{S}$ phase.

Understanding how eukaryotic cells use a large number of inefficient origins to ensure the timely duplication of their genome is a challenging issue. Indeed, population-based replication assays generate averaged replication profiles and are therefore unable to assess cell-to-cell variations. Recently, a new generation of single-molecule assays has been developed to monitor DNA replication at the level of individual chromosomes. In this review, we discuss how these new assays have modified our view of replication dynamics and origin efficiency in eukaryotic cells, with a special emphasis on the yeast $S$. cerevisiae.

\section{DNA replication programs: the yeast paradigm}

Replication origins are best characterized in $S$. cerevisiae. They were first identified as small genetic elements called autonomous replication sequence (ARS), which allow the maintenance of minichromosomes (Newlon and Theis 1993). ARS elements contain a perfect match to an 11-bp ARS consensus sequence that is recognized by ORC to recruit pre-RC components. The first direct evidence that ARS elements act as replication origins was brought by two-dimensional (2D) gel analysis of plasmid DNA replication (Brewer and Fangman 1987). However, only a fraction of these ARS elements were shown to act as chromosomal origins in their natural context (Newlon and Theis 1993). Importantly, the ability of 2D gel assays to discriminate between active (bubble arc) and passive replication (Y arc) revealed that most of the yeast origins are intrinsically inefficient, as they 
fire in only a fraction of the cell cycles. Later on, extensive analyses of chromosomes III and VI by 2D gel electrophoresis confirmed that the budding yeast genome contains an excess of replication origins (Friedman et al. 1997; Yamashita et al. 1997). As for other eukaryotes, $S$. cerevisiae origins vary both in their timing and efficiency of initiation.

The availability of the complete sequence of the $S$. cerevisiae genome and the development of microarray-based technologies are at the origin of the first genome-wide analysis of a eukaryotic DNA replication program (Raghuraman et al. 2001). Using a density transfer approach to isolate newly replicated sequences, Raghuraman and colleagues identified 300 prominent peaks on genome-wide profiles corresponding to replication origins. Following on this pioneering study, other genome-wide approaches were developed. A study based on DNA copy number changes at origins identified $\sim 250$ yeast origins, among which $\sim 100$ were repressed by the DNA replication checkpoint (Yabuki et al. 2002). Another study took advantage of the presence of singlestranded DNA at replication forks to map replication origins in budding yeast and in fission yeast (Feng et al. 2007). Finally, a comparative analysis of phylogenetic conservation of sequences between five related Saccharomyces species was used to map $S$. cerevisiae origins at base pair resolution (Nieduszynski et al. 2006). These origins appear in OriDB, a web-based catalogue gathering all the genome-wide datasets available for S. cerevisiae (Nieduszynski et al. 2007).

Data generated by microarray-based assays support the view that initiation of DNA replication follows a temporal program that is imposed by epigenetic mechanisms and is stably transmitted to daughter cells (Knott et al. 2009; Raghuraman et al. 2001). However, this model has been recently challenged by Shao and colleagues, who showed that all yeast chromosomes VI have different replication profile when analyzed at the singlemolecule level (Czajkowsky et al. 2008). These authors also reported that single-molecule profiles recapitulate microarray data when averaged, suggesting that predetermined replication programs are artifactually generated in population-based assays. Whether replication timing is deterministic as indicated by microarray-based data or probabilistic, as suggested by the Shao study, is an important question that is addressed in the following sections.

\section{Single-molecule analysis of DNA replication profiles}

Single-molecule assays such as electron microscopy (EM) and DNA fiber autoradiography are at the origin of paradigms such as replication bubbles and bidirectional replication from irregularly spaced origins (Huberman and Riggs 1966; Taylor and Miner 1968). EM allows the direct visualization of branched replication intermediates on individual molecules spread over a carbon-coated grid. This technique was used to map the first eukaryotic origin of DNA replication (Bozzoni et al. 1981) and is still used to monitor pathological transitions at replication fork (Lopes et al. 2006). Fiber autoradiography is based on the pulse labeling of chromosomes with tritiated thymidine and on the detection of newly replicated regions by autoradiography (Huberman and Riggs 1966). DNA fiber autoradiography provided the first quantitative assessment of origin densities and replication fork rates in metazoan genomes. Nowadays, this time-consuming assay has been replaced with fiber fluorography approaches, in which newly replicated DNA is substituted with halogenated nucleotide derivatives, such as bromo- $(\mathrm{Br})$, chloro(Cl), or iododeoxyuridine (IdU) and is visualized by indirect immunofluorescence using specific antibodies (Jackson and Pombo 1998).

Different versions of DNA fiber fluorographic assays have been developed to monitor the replication of individual DNA molecules. These techniques differ essentially on the way DNA fibers are stretched on glass surfaces and how ongoing replication is labeled (Fig. 1). The most commonly used assays include DNA spreads (Jackson and Pombo 1998), DNA combing (Michalet et al. 1997), and SMARD (Norio and Schildkraut 2001). DNA fibers can also be stretched by capillarity in microchannels generated with nanofabrication processes (Sidorova et al. 2008). All these approaches can be coupled to fluorescence in situ hybridization (FISH) to identify regions of interest and monitor DNA replication at specific loci (Lebofsky et al. 2006; Norio and Schildkraut 2001; Pasero et al. 2002).

$S$. cerevisiae is an ideal system to monitor replication profiles at the single-molecule level. Its replication origins have been mapped, cell cultures can be easily synchronized in S phase, and multiple mutations affecting DNA replication are available. 


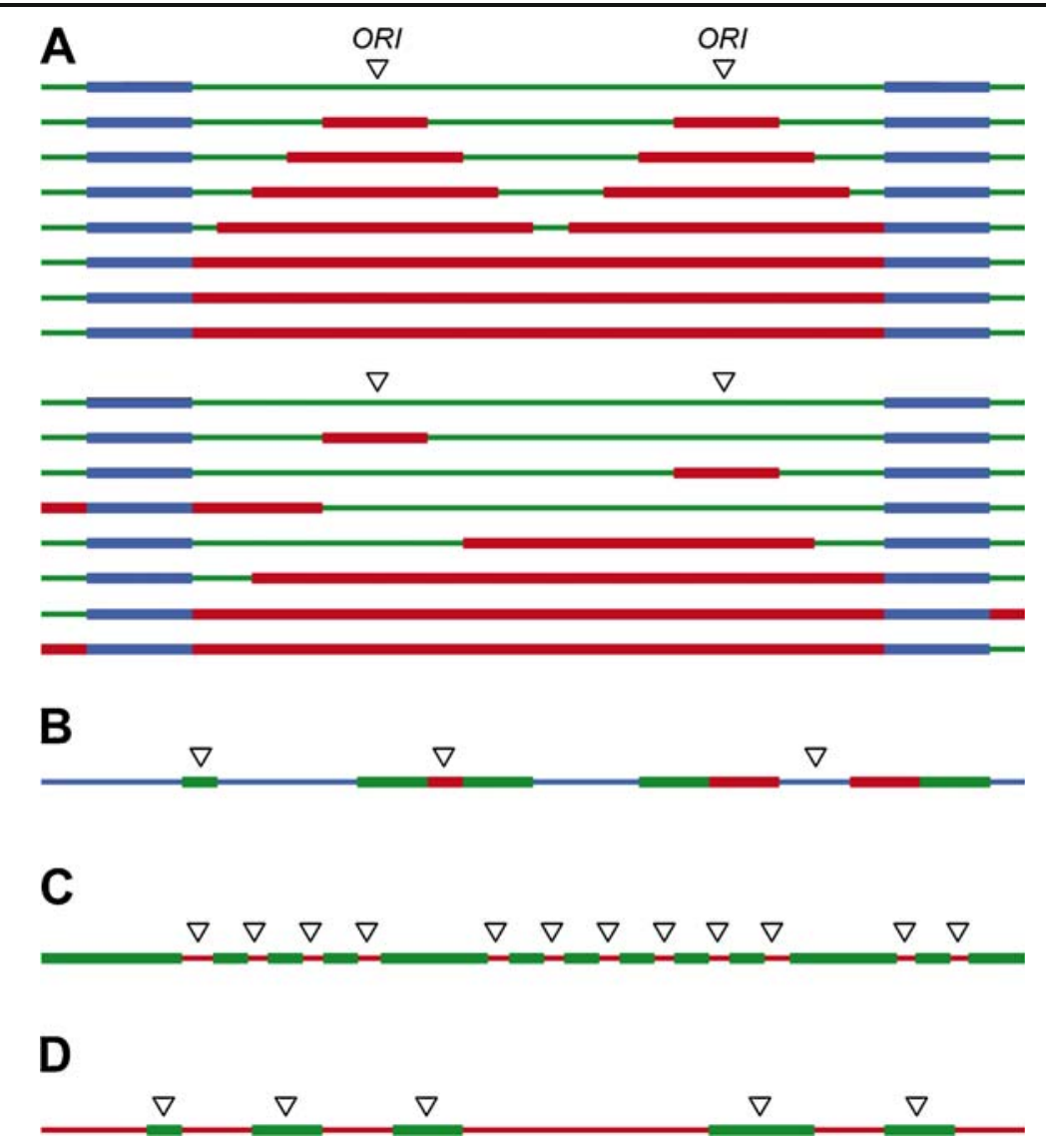

Fig. 1 Strategies used to monitor origin use with singlemolecule assays. a Single-molecule analysis of replicated DNA (SMARD). In this assay, ongoing DNA replication is labeled with two successive pulses of halogenated nucleotides (green/ red) in an asynchronous population of cells. Regions of interest are then enriched by pulsed-field gel electrophoresis, spread on silanized coverslips, and replicated fibers are assembled relative to FISH probes. Ordered patterns (top) are indicative of efficient origin usage, whereas non-ordered patterns (bottom) reflect poor origin efficiency (Norio and Schildkraut 2001); b double labeling of ongoing replication with short pulses of IdU/ CldU (Jackson and Pombo 1998). In this approach, an asynchronous population of cells is pulsed-labeled for a short period of time with halogenated nucleotides to determine the

However, budding yeast cells lack a thymidine kinase gene (TK) and must be genetically engineered to incorporate bromo-deoxyuridine (BrdU). Using TK + cells, Schwob and colleagues were able to detect BrdU incorporation at approximately 190 replication origins scattered every $46 \mathrm{~kb}$ on average in the yeast genome (Lengronne et al. 2001). This value is significantly larger than the $35-\mathrm{kb}$ interorigin distance estimated earlier from 2D gel and microarray-based analyses (Newlon and Theis 1993; position of replication origins (arrowheads). DNA fibers can be counterstained with anti-ssDNA antibodies (blue) (Versini et al. 2003), and specific regions can be analyzed by FISH (Lebofsky et al. 2006); c DNA combing analysis of densely spaced origins in Xenopus egg extracts (Marheineke and Hyrien 2001). Initiation sites are labeled with DIG-dUTP (red) to mark replication origins (arrowheads) after release from an aphidicolin block and biotin-dUTP (green) is added at variable times until completion of DNA replication; $\mathbf{d}$ DNA combing analysis of interorigin distances (IODs) in yeast. Cells are synchronized in $G_{1}$ with $\alpha$-factor and are released into $S$ phase in the presence of $\mathrm{BrdU}$ (green) to label origins (arrowheads) and hydroxyurea (HU) to block elongation. DNA is counterstained with anti-ssDNA antibodies (red)

Raghuraman et al. 2001), supporting the view that only a fraction of the replication origins fire in a given cell. These authors were also able to reveal subtle initiation defects in cells defective for the CDK inhibitor Sic1, which were hardly detectable with classical biochemical assays (Lengronne and Schwob 2002). Stronger initiation defects were also measured in various initiation mutants using this approach (Devault et al. 2002; Semple et al. 2006; Shimada et al. 2002). 
Another advantage of single-molecule assays is their ability to map replication origins in repetitive sequences. This is best illustrated with the yeast ribosomal DNA (rDNA) array, which consists in 100200 identical copies of a 9.1-kb repeat containing an origin of replication. $2 \mathrm{D}$ gel assays indicate that only $20 \%$ of these origins are active on the chromosome, but the position of initiation sites within the rDNA array could not be addressed due to the repetitive nature of this material. DNA combing studies revealed that active origins form groups of two to five adjacent units and that clusters of active origins are separated from each other with unreplicated regions of variable length (Fig. 2a). Initiation within these large gaps is repressed by the histone deacetylase Sir2, which promotes heterochromatin formation at telomeres and silent mating loci (Pasero et al. 2002). This epigenetic regulation of origin usage at the rDNA is not maintained over cell divisions, different groups of rDNA origins being used in a clonal population of cells (Fig. 2b).

\section{Single-molecule analysis: lessons from yeast}

Single-molecule assays have proven powerful approaches to monitor DNA replication in yeast. However, several important parameters must be considered in order to avoid experimental biases. Some of these issues are illustrated in Fig. 2a, which shows representative DNA fibers encompassing the junction between rDNA and single-copy sequences on chromosome XII. Active origins form clusters in the rDNA array, whereas initiation sites are more dispersed in single-copy regions of the genome. Active origins can be easily assigned to specific rDNA units owing to the high FISH probes coverage at this locus (one probe $/ 9.1 \mathrm{~kb}$; Fig. 2b). In contrast, matching BrdU tracks with annotated origins is much more difficult for single-copy sequences, especially when BrdU signals are located at a large distance from FISH probes. For instance, while BrdU tracks are clearly centered on early origins ARS1211 and ARS1213 on the DNA fiber 3, determining which origins are associated to replication tracks on fiber 6 is less obvious (Fig. 2a). This uncertainty is due in part to the fact that $\mathrm{BrdU}$ signals are frequently interrupted. Another potential source of error comes from local variations in DNA fiber extension. In theory, combed DNA fibers show a constant stretching of $2 \mathrm{~kb} / \mu \mathrm{m}$ (Michalet et al. 1997). However, locally compressed or overextended zones can be detected along DNA fibers, as illustrated in Figs. $2 \mathrm{~d}$ and e. These local variations decrease the resolution of DNA fiber assays proportionally to the distance between FISH probes. Consequently, the $1-\mathrm{kb}$ resolution put forward in some DNA fiber studies is usually largely overestimated.

Besides origin mapping, single-molecule approaches are frequently used to measure the density of initiation events along individual chromosomes at the genomewide level (Lengronne et al. 2001). In a typical experiment, cells are arrested in $G_{1}$ and released synchronously into $\mathrm{S}$ phase in the presence of BrdU to label newly replicated DNA and of hydroxyurea (HU) to block elongation. The rate of initiation is expressed as the mean center-to-center distance between BrdU tracks. As shown in Fig. 3a, interorigin distances (IODs) are influenced by the overall length of analyzed DNA fibers. In initial DNA combing experiments, fiber lengths could not be determined for technical reasons, and IODs were essentially measured within clusters of early origins (Lengronne et al. 2001; Lengronne and Schwob 2002; Shimada et al. 2002). With the development of single-stranded DNA (ssDNA) staining procedures, BrdU tracks could be assigned to the same fiber over much longer distances, revealing the presence of large repressed domains in between clusters of active origins (Tourriere et al. 2005). IODs measured after counterstaining of DNA fibers are $25 \%$ larger for the same cells (Fig. 3b), stressing the importance of analyzing replication profiles on DNA fibers of comparable lengths.

When properly controlled, DNA fiber assays are able to detect slight modifications in origin efficiency. This is illustrated in Fig. 3c, where subtle changes in initiation rates are detected in thermosensitive orc 2-1 mutants grown at different temperature (PP, unpublished results). However, it is important to note that initiation rates can be overestimated when IODs are measured in cells presenting severe initiation defects. For instance, depletion of the Orc6 subunit in GALORC6 cells increases IODs by $70 \%$ (Semple et al. 2006), but this analysis does not take into account DNA fibers containing a single initiation event (Fig. 3d). To avoid this problem, initiation rates can also be expressed as the ratio of the number of active origins to the total length of DNA fibers (Fig. 3e). 
The examples presented above indicate that singlemolecule assays represent powerful approaches to monitor initiation rates in different cell types or growth conditions. Combined with FISH, these approaches can also measure origin efficiency at specific regions (Czajkowsky et al. 2008; Pasero et al. 2002). However, these studies are usually restricted to a small portion of the yeast genome [less than $2 \%$ for chromosome VI (Czajkowsky et al. 2008)]. To estimate the fraction of replication origins that are used during two successive cell cycles on a genome-wide basis, we used a different approach in which origins are labeled with halogenated nucleotides in two successive cell cycles before DNA combing analysis (V. Cordon-Preciado and PP; unpublished data). To this end, wild-type cells were arrested in $\mathrm{G}_{1}$ and were released synchronously into $S$ phase in the presence of IdU (red) and were arrested with HU. After $90 \mathrm{~min}$, IdU was washed away, and cells were released from HU. Cells were then arrested in $G_{1}$ of the next cell cycle and were further released into $\mathrm{S}$ phase for another $90 \mathrm{~min}$ in the presence of CldU (green) and HU. DNA fibers were stretched by DNA combing and halogenated nucleotides were detected with specific antibodies as described (Tourriere et al. 2005). As shown in Fig. 4, we found that less than $40 \%$ of the replication origins fire twice in two successive cell cycles. Many adjacent or overlapping signals were also detected, reflecting origin interference between neighboring origins. Together, these profiles support the view that cells use different sets of origins to replicate their chromosomes. Flexible patterns of origin usage were also detected along purified rDNA fibers (Fig. 4c), which is consistent with earlier studies (Pasero et al. 2002).

DNA fiber studies indicate that the yeast genome contains a large excess of replication origins and that only a fraction of these are used during a given cell cycle. Importantly, DNA combing studies also revealed the presence of large inactive regions in HU-treated cells (Tourriere et al. 2005; Versini et al. 2003). These domains are absent in checkpoint mutants, indicating that they correspond to latereplicating regions repressed by checkpoint kinases (Tourriere et al. 2005). Although origin usage is extremely flexible within early replication domains (Fig. 4), the detection of late domains along individual DNA fibers is consistent with microarray data and argues against the probabilistic model proposed by Shao and colleagues (Czajkowsky et al. 2008).
Fig. 2 DNA combing analysis of origin efficiency at the yeast rDNA array. Replication origins are labeled with BrdU in early $\mathrm{S}$ phase as indicated in Fig. 1d; a representative images of chromosome XII fragments centered on the junction between the yeast rDNA array and single-copy sequences. Short $(3 \mathrm{~kb})$ FISH probes were used to label rRNA genes. The left edge of the rDNA array was positioned relative to a $10-\mathrm{kb}$ FISH probe (Pasero et al. 2002). Green BrdU. Red FISH probes. The position of early $(E)$ and late $(L)$ origins is indicated; b schematic representation of the distribution of active (filled circles) and inactive (open circles) rDNA origins for ten rDNA fibers encompassing 22 rDNA units (Pasero et al. 2002). The frequency of origin use is indicated for each origin; c distribution of center-to-center distances measured between adjacent rDNA probes $(n=1,400)$; d, e mean distance between FISH rDNA probes calculated with a sliding window encompassing ten rDNA repeats. Scale bar $100 \mathrm{~kb}$

What is the functional relevance of late replication domains in yeast genome? Indirect evidence derived from the analysis of $m r c l \Delta$ and tofl $\Delta$ mutants suggests that these domains play an important role in the cellular response to replication stress. Mrc1 and Tof1 form a complex with Csm3 at the replication fork and have been implicated in the coordination between replicative helicases and polymerases (Katou et al. 2003). When $\operatorname{mrcl} \Delta$ and tofl $\Delta$ mutants are exposed to HU, replisome components uncouple from sites of DNA synthesis (Katou et al. 2003) and forks are unable to restart after release from HU (Tourriere et al. 2005). Yet, tofl $\Delta$ mutants are much less sensitive to HU than $m r c l \Delta$ cells. As shown in Fig. $3 b$, this is due to the fact that, unlike $m r c 1 \Delta$ mutants, tof $1 \Delta$ cells are still partially able to repress late origins in $\mathrm{HU}$, which can be used to complete $\mathrm{S}$ phase upon release from the drug (Tourriere et al. 2005). These data indicate that $S$. cerevisiae cells rely both on origin redundancy and replication timing control to deal with replication stress. Whether this is also the case for higher eukaryotes is discussed in the following section.

\section{DNA fiber analyses of metazoan replication programs}

Pioneering DNA fiber studies from Jackson and Pombo have shown that groups of replicons fire synchronously in HeLa cells at specific times in $\mathrm{S}$ phase (Jackson and Pombo 1998). Using an elegant Genomic Morse Code approach covering a $1.5-\mathrm{Mb}$ region of human chromosome 14, Bensimon and 


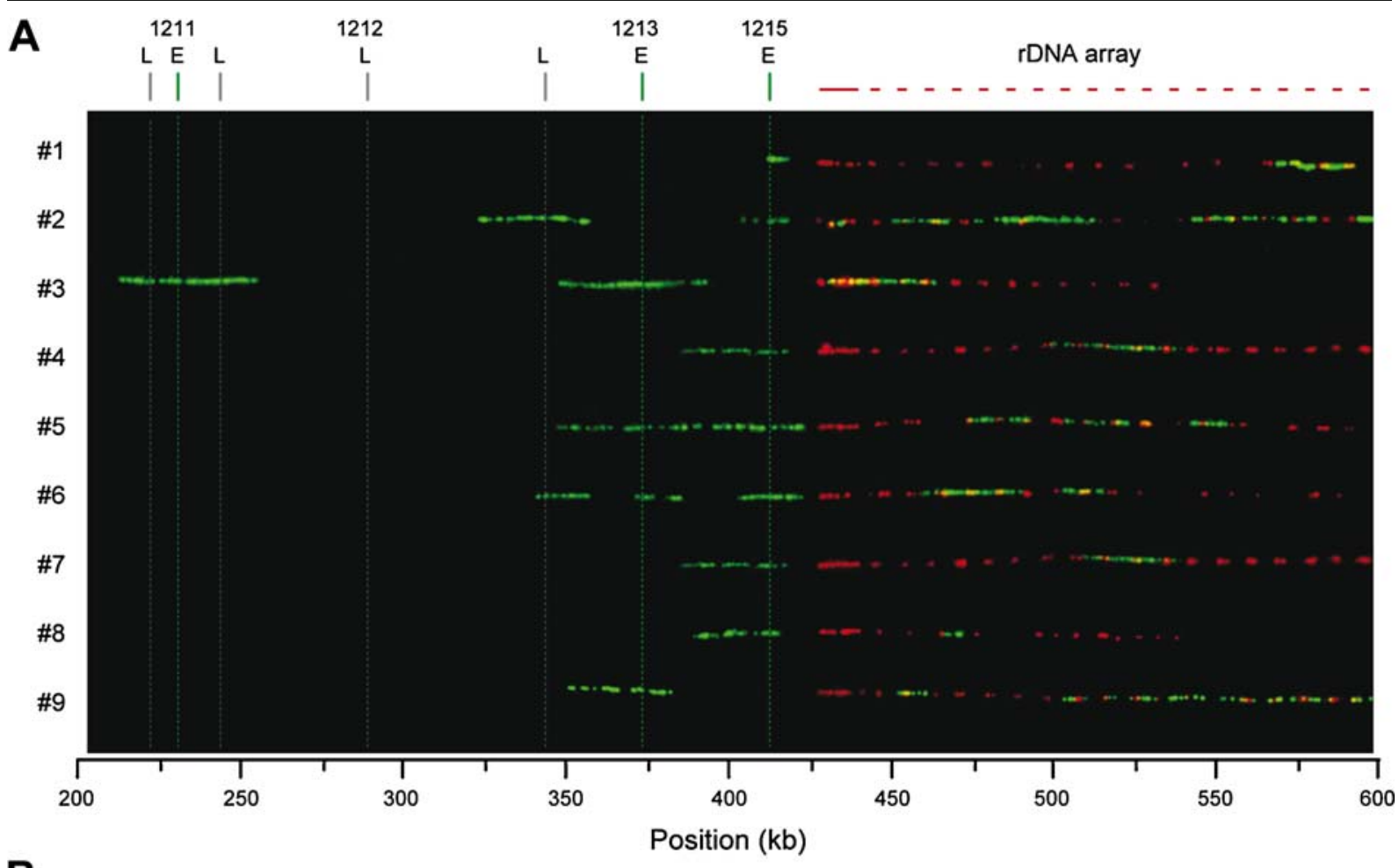

B

rDNA origins : $1 \begin{array}{llllllllllllllllllllll}1 & 2 & 3 & 4 & 5 & 6 & 7 & 8 & 9 & 10 & 11 & 12 & 13 & 14 & 15 & 16 & 17 & 18 & 19 & 20 & 21 & 22\end{array}$

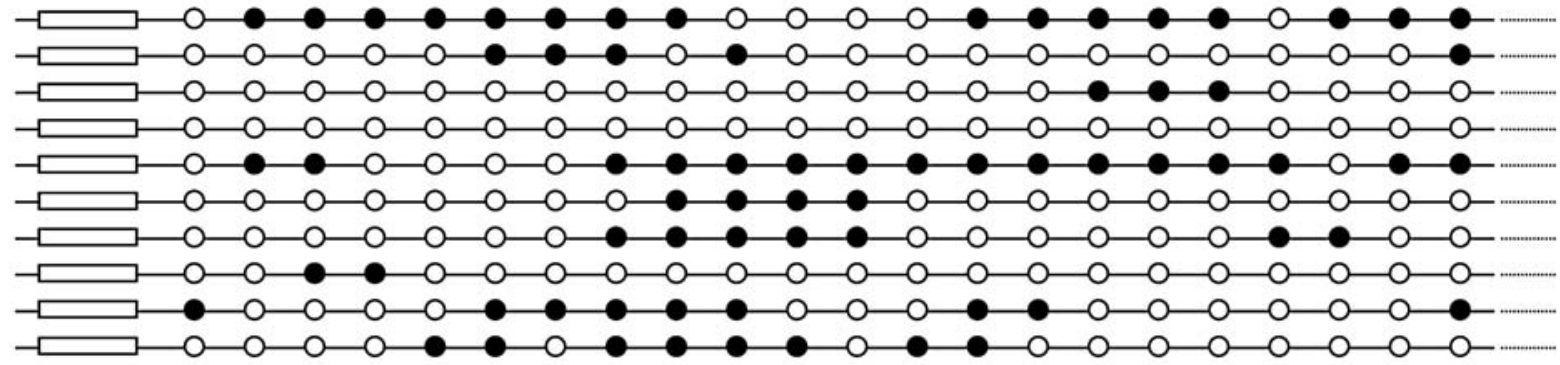

$\begin{array}{lllllllllllllllllllllll}\text { Frequency: } & 0.1 & 0.2 & 0.3 & 0.2 & 0.2 & 0.4 & 0.3 & 0.6 & 0.6 & 0.6 & 0.4 & 0.3 & 0.2 & 0.4 & 0.3 & 0.3 & 0.3 & 0.3 & 0.2 & 0.2 & 0.2 & 0.4\end{array}$

C

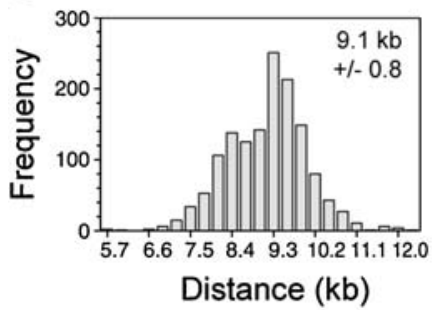

D

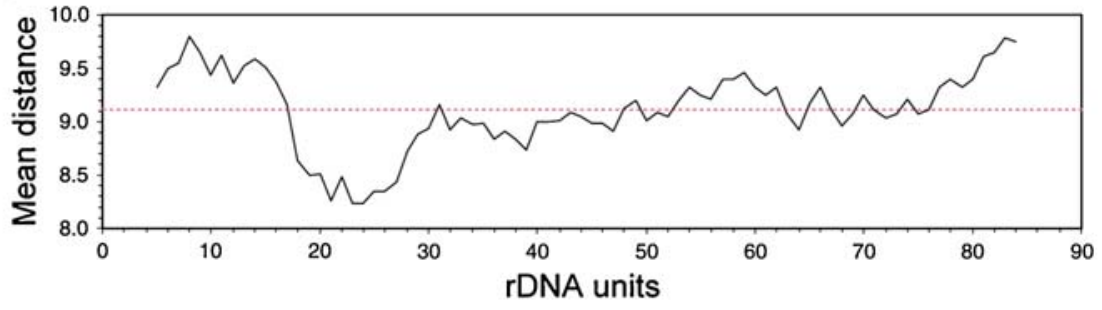

E 
A

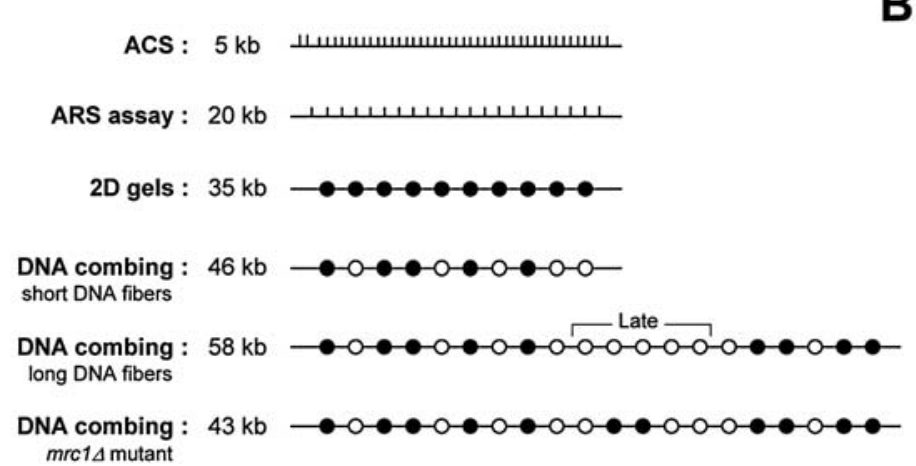

B

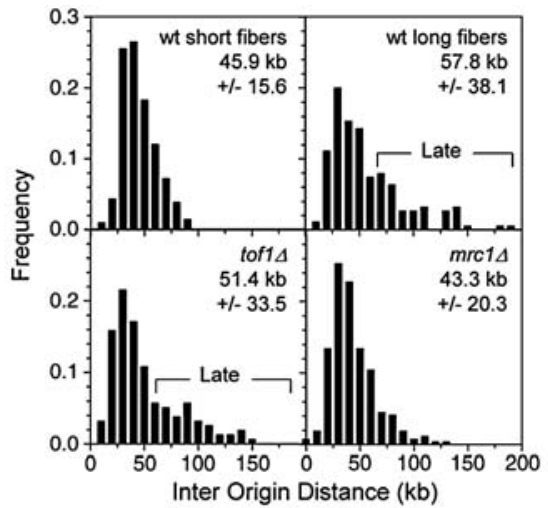

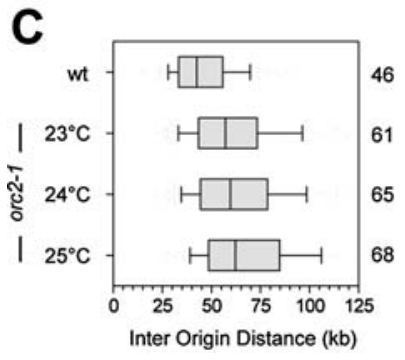

D

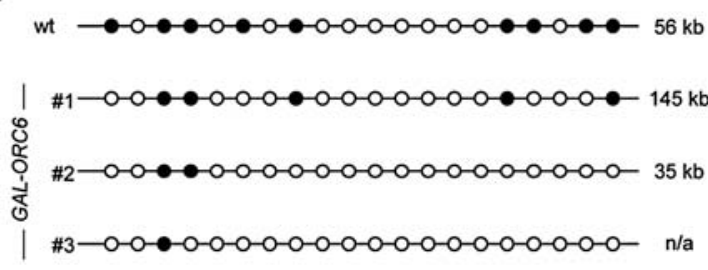

E

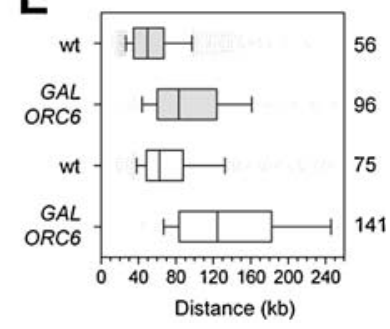

Fig. 3 DNA combing analysis of origin efficiency at singlecopy loci; a distribution of replication origins in the $S$. cerevisiae genome. The yeast genome contains over 12,000 matches to the ARS consensus sequence, but only a fraction of these elements show ARS activity on a plasmid (1/20 kb). 2D gel analyses and microarray data show that only a subset of these ARS elements is active on the chromosome $(1 / 35 \mathrm{~kb})$ (Newlon and Theis 1993; Raghuraman et al. 2001). DNA combing analyses indicate that only a fraction of chromosomal origins are active in a given cell $(1 / 46 \mathrm{~kb})$ (Lengronne et al. 2001; Lengronne and Schwob 2002; Shimada et al. 2002). Analysis of larger DNA fibers by DNA combing reveals the presence of large unreplicated domains containing late origins, increasing again the average IOD $(1 / 58 \mathrm{~kb})$. Derepression of

colleagues revealed that replication origins form clusters, which are distributed every $40 \mathrm{~kb}$ on average and are reminiscent of the "initiation zones" detected earlier with biochemical approaches (DePamphilis 1999). Only one third of these clusters fire every cell cycle, presumably because of origin interference within and between initiation zones (Lebofsky et al. 2006). A similar analysis of DNA replication at the human rDNA array revealed a complex pattern of initiation events and replication pause sites (Lebofsky and Bensimon 2005). Replication profiles change as cells differentiate, concomitantly with changes in chromatin structure and transcriptional activity (Norio et al. 2005; Hiratani et al. 2008).

DNA fiber analyses also revealed that initiation sites are irregularly distributed during early Xenopus late domains in the mrcl $\Delta$ mutant restores shorter IODs $(43 \mathrm{~kb})$; b distribution of IODs in wild type (short and long fibers), mrcl $\Delta$ and tofl $\Delta$ cells (Tourriere et al. 2005); c distribution of IODs in wild type and orc2-1 cells grown at the indicated temperatures. Boxes 25-75 percentile range. Whiskers 10-90 percentile range. Medians are indicated in kilobases; d schematic representation of the distribution of active origins (filled circles) in GAL-ORC6 cells upon Orc6 depletion. IODs are indicated in kilobases; e comparison of IODs (filled boxes) and the ratio of origins to total DNA length (empty boxes) measured in wild type and GAL-ORC6 cells (Semple et al. 2006). Boxes $25-75$ percentile range. Whiskers $10-90$ percentile range. Medians are indicated in kilobases

development and are activated at different times throughout S phase (Blow et al. 2001; Herrick et al. 2000). Interestingly, replication dynamics in Xenopus egg extracts is modulated by the ATR checkpoint kinase even in the absence of exogenous replication stress (Marheineke and Hyrien 2004; Shechter et al. 2004). Using a similar approach to the one described in Fig. 4, Marheineke and colleagues also revealed that replication timing control in Xenopus embryos is deterministic at the level of chromosomal domains but is probabilistic at the level of individual origins (Labit et al. 2008), which is consistent with yeast data. Together, these data indicate that a large excess of functional pre-RCs are assembled on chromatin in Xenopus egg extracts but that the vast majority of these potential replication origins are not used during 


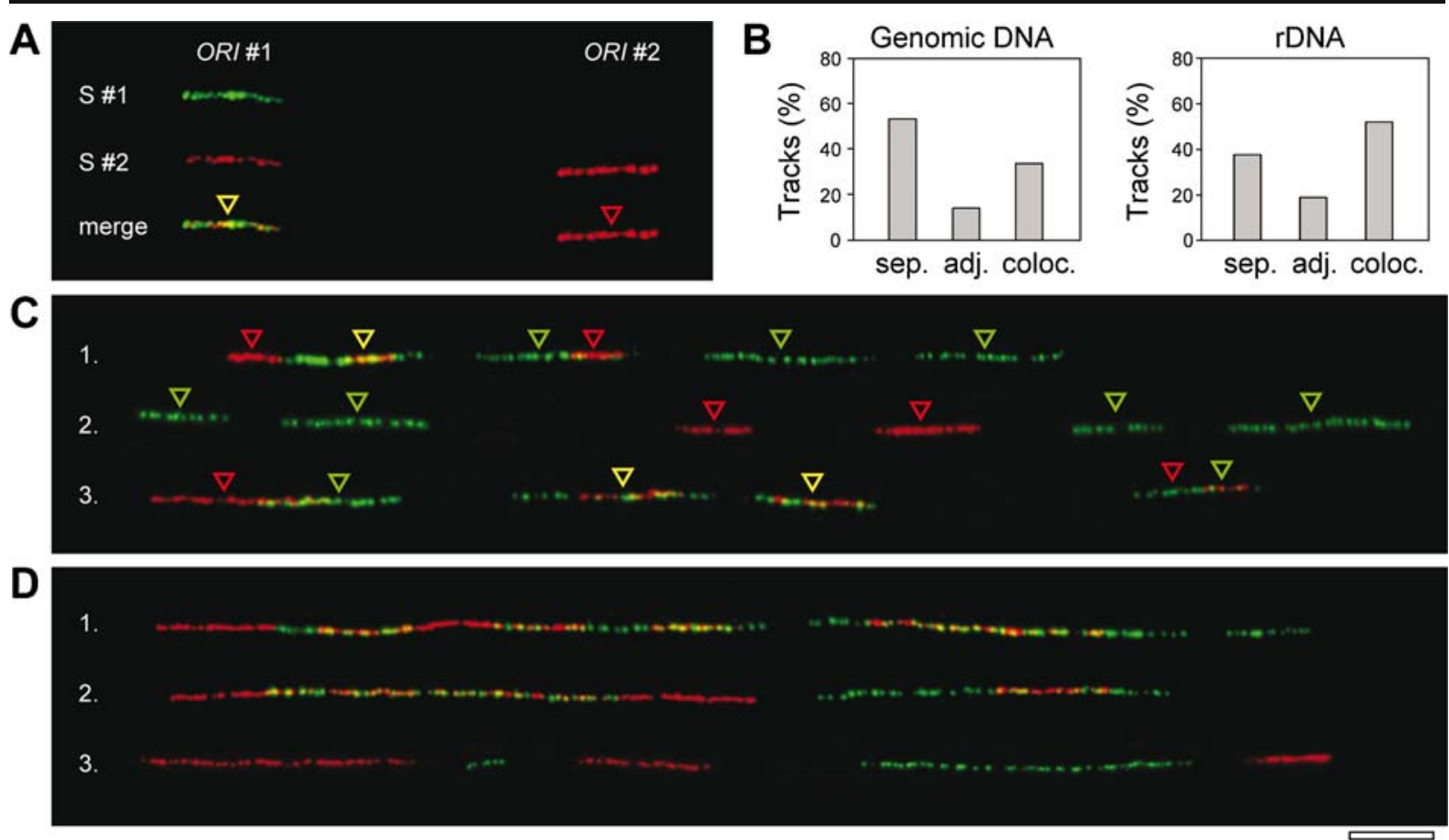

Fig. 4 DNA combing analysis of yeast origins active in two successive cell cycles; a early origins were sequentially labeled with CldU (green) in the first cell cycle and with IdU (red) in the second cell cycle, as described in the text. This example shows that ORI\#I was used in both cell cycles, whereas ORI\#2 fired only during the second $\mathrm{S}$ phase; $\mathbf{b}$ percentage of separate (sep.), adjacent (adj.), and overlapping (coloc.) IdU and CldU

an unperturbed S phase. In replication stress situation, however, these dormant origins can be mobilized to complete genome replication (Woodward et al. 2006).

The replication program of differentiated cells can be artificially remodeled into an early development replication profile by incubating nuclei into mitotic Xenopus egg extracts. This reprogramming depends on the reorganization of chromosomal loop domains in a topoisomerase II-dependent manner (Lemaitre et al. 2005). As reported by Taylor more than 30 years ago, similar reprogramming processes also operate in mammalian cells in response to environmental changes (Taylor 1977). By manipulating nucleotide pools in CHO cells, Taylor detected a negative correlation between the rate of fork progression and the frequency of initiation, indicating that cells are able to activate additional origins to compensate for slow forks (Gilbert 2007; Taylor 1977). These results were recently confirmed by the Debatisse lab, which showed that the AMPD2 locus is replicated from a single origin when forks progress at a normal rate, signals detected in single-copy sequences and at the rDNA locus; $\mathbf{c}$ representative DNA fibers showing origins firing in the first cell cycle (green arrowheads), second cell cycle (red arrowheads), or both cycles (yellow arrowheads); d origin usage at the rDNA array. Ribosomal DNA fibers were isolated as described previously (Pasero et al. 2002). Scale bar $20 \mathrm{~kb}$

whereas additional origins fire at this locus when forks progression is slowed down (Anglana et al. 2003). Moreover, Debatisse and colleagues revealed that this adaptation correlates with changes in the organization of chromatin loops (Courbet et al. 2008), as is the case for mitotic reprogramming events in Xenopus egg extracts (Lemaitre et al. 2005).

A massive activation of dormant origins has also been reported in various situations affecting fork progression. These include overexpression of translesion DNA polymerases (Pillaire et al. 2007), the depletion of CHK1 (Maya-Mendoza et al. 2007), BLM (Rao et al. 2007), HR proteins (Daboussi et al. 2008), and topoisomerase I (Tuduri et al. 2009) and exposure to HU (Ge et al. 2007; Ibarra et al. 2008). Importantly, the latter studies also revealed that dormant origins are dispensable for normal growth but are essential for viability under replication stress (Ge et al. 2007; Ibarra et al. 2008).

Several non-exclusive models have been proposed to explain the homeostatic coordination of fork rates 
and replicon sizes (Blow and Ge 2009; Gilbert 2007; Herrick and Bensimon 2008). The simplest of these models stipulates that, in normal growth conditions, dormant origins are passively replicated by forks progressing from the closest active origins. When fork progression is impaired, dormant origins would have more time to fire, and the initiation rate would automatically increase. The activation of dormant origins in HU-exposed cells is paradoxical, since stalled forks are known to elicit a replication stress response preventing further initiation (Tourriere and Pasero 2007). However, it has been reported that dormant origins fire only in the vicinity of stalled forks but are repressed at late-replicating regions (Ge et al. 2007). How eukaryotic cells differentiate regions with ongoing replication from inactive domains is currently unclear.

In conclusion, DNA fiber assays have profoundly modified our understanding of DNA replication programs. They have also revealed the importance of dormant origins in the maintenance of genome integrity during $\mathrm{S}$ phase. The picture emerging from these studies indicates that replication programs are highly flexible in eukaryotic cells and are controlled by a complex interplay between epigenetic mechanisms and checkpoint pathways. High-resolution maps of the distribution of replication origins in the human genome should be available soon. Combined with single-molecule assays, genome-wide data will undoubtedly bring new light on the mechanisms by which normal cells adapt their replication profiles to environmental changes and how these mechanisms are hijacked by cancer cells to resist to genotoxic drugs targeting replication forks.

Acknowledgments We thank Laure Crabbe, Armelle Lengronne, Susanne Schmidt, and Etienne Schwob for helpful comments and discussions. We also thank Etienne Schwob and the DNA combing facility of Montpellier for silanized coverslips. Work in the Pasero lab is supported by ANR, INCa and by the Fondation pour la Recherche Médicale (Equipe FRM). ST also thanks CNRS for support.

\section{References}

Anglana M, Apiou F, Bensimon A, Debatisse M (2003) Dynamics of DNA replication in mammalian somatic cells: nucleotide pool modulates origin choice and interorigin spacing. Cell 114:385-394
Arias EE, Walter JC (2007) Strength in numbers: preventing rereplication via multiple mechanisms in eukaryotic cells. Genes Dev 21:497-518

Azvolinsky A, Giresi PG, Lieb JD, Zakian VA (2009) Highly transcribed RNA polymerase II genes are impediments to replication fork progression in Saccharomyces cerevisiae. Mol Cell 34:722-734

Bell SP, Dutta A (2002) DNA replication in eukaryotic cells. Annu Rev Biochem 71:333-374

Blow JJ, Ge XQ (2009) A model for DNA replication showing how dormant origins safeguard against replication fork failure. EMBO Rep 10:406-412

Blow JJ, Gillespie PJ, Francis D, Jackson DA (2001) Replication origins in Xenopus egg extract Are 5-15 kilobases apart and are activated in clusters that fire at different times. J Cell Biol 152:15-25

Bozzoni I, Baldari CT, Amaldi F, Buongiorno-Nardelli M (1981) Replication of ribosomal DNA in Xenopus laevis. Eur J Biochem 118:585-590

Brewer BJ, Fangman WL (1987) The localization of replication origins on ARS plasmids in S. cerevisiae. Cell 51:463-471

Courbet S, Gay S, Arnoult N, Wronka G, Anglana M, Brison O, Debatisse M (2008) Replication fork movement drives remodeling of chromatin loops and origin choice in mammalian cells. Nature 455:557-560

Czajkowsky DM, Liu J, Hamlin JL, Shao Z (2008) DNA combing reveals intrinsic temporal disorder in the replication of yeast chromosome VI. J Mol Biol 375:12-19

Daboussi F, Courbet S, Benhamou S, Kannouche P, Zdzienicka MZ, Debatisse M, Lopez BS (2008) A homologous recombination defect affects replication-fork progression in mammalian cells. J Cell Sci 121:162-166

DePamphilis ML (1999) Replication origins in metazoan chromosomes: fact or fiction? Bioessays 21:5-16

Devault A, Vallen EA, Yuan T, Green S, Bensimon A, Schwob E (2002) Identification of Tah11/Sid2 as the ortholog of the replication licensing factor Cdt1 in Saccharomyces cerevisiae. Curr Biol 12:689-694

Feng W, Raghuraman MK, Brewer BJ (2007) Mapping yeast origins of replication via single-stranded DNA detection. Methods 41:151-157

Friedman KL, Brewer BJ, Fangman WL (1997) Replication profile of Saccharomyces cerevisiae chromosome VI. Genes Cells 2:667-678

Gambus A, Jones RC, Sanchez-Diaz A, Kanemaki M, van Deursen F, Edmondson RD, Labib K (2006) GINS maintains association of $\mathrm{Cdc} 45$ with $\mathrm{MCM}$ in replisome progression complexes at eukaryotic DNA replication forks. Nat Cell Biol 8:358-366

Ge XQ, Jackson DA, Blow JJ (2007) Dormant origins licensed by excess Mcm 27 are required for human cells to survive replicative stress. Genes Dev 21:3331-3341

Gilbert DM (2007) Replication origin plasticity, Taylor-made: inhibition vs recruitment of origins under conditions of replication stress. Chromosoma 116:341-347

Herrick J, Bensimon A (2008) Global regulation of genome duplication in eukaryotes: an overview from the epifluorescence microscope. Chromosoma 117:243-260

Herrick J, Stanislawski P, Hyrien O, Bensimon A (2000) Replication fork density increases during DNA synthesis in X. laevis egg extracts. J Mol Biol 300:1133-1142 
Hiratani I, Ryba T, Itoh M, Yokochi T, Schwaiger M, Chang CW, Lyou Y, Townes TM, Schubeler D, Gilbert DM (2008) Global reorganization of replication domains during embryonic stem cell differentiation. PLoS Biol 6: e245

Hiratani I, Takebayashi S-i LuJ, Gilbert DM (2009) Replication timing and transcriptional control: beyond cause and effect-part II. Curr Opin Genet Dev 19:142-149

Huberman JA, Riggs AD (1966) Autoradiography of chromosomal DNA fibers from Chinese hamster cells. PNAS 55:599-606

Ibarra A, Schwob E, Mendez J (2008) Excess MCM proteins protect human cells from replicative stress by licensing backup origins of replication. PNAS 105:8956-8961

Jackson DA, Pombo A (1998) Replicon clusters are stable units of chromosome structure: evidence that nuclear organization contributes to the efficient activation and propagation of S phase in human cells. J Cell Biol 140:1285-1295

Katou Y, Kanoh Y, Bando M, Noguchi H, Tanaka H, Ashikari T, Sugimoto K, Shirahige K (2003) S-phase checkpoint proteins Tof1 and Mrc1 form a stable replication-pausing complex. Nature 424:1078-1083

Knott SR, Viggiani CJ, Tavare S, Aparicio OM (2009) Genome-wide replication profiles indicate an expansive role for $\mathrm{Rpd} 3 \mathrm{~L}$ in regulating replication initiation timing or efficiency, and reveal genomic loci of Rpd3 function in Saccharomyces cerevisiae. Genes Dev 23:1077-1090

Labit H, Perewoska I, Germe T, Hyrien O, Marheineke K (2008) DNA replication timing is deterministic at the level of chromosomal domains but stochastic at the level of replicons in Xenopus egg extracts. Nucleic Acids Res 36:5623-5634

Lebofsky R, Bensimon A (2005) DNA replication origin plasticity and perturbed fork progression in human inverted repeats. Mol Cell Biol 25:6789-6797

Lebofsky R, Heilig R, Sonnleitner M, Weissenbach J, Bensimon A (2006) DNA replication origin interference increases the spacing between initiation events in human cells. Mol Biol Cell 17:5337-5345

Lemaitre JM, Danis E, Pasero P, Vassetzky Y, Mechali M (2005) Mitotic remodeling of the replicon and chromosome structure. Cell 123:787-801

Lengronne A, Schwob E (2002) The yeast CDK inhibitor Sic1 prevents genomic instability by promoting replication origin licensing in late G(1). Mol Cell 9:1067-1078

Lengronne A, Pasero P, Bensimon A, Schwob E (2001) Monitoring $\mathrm{S}$ phase progression globally and locally using BrdU incorporation in $\mathrm{TK}(+)$ yeast strains. Nucleic Acids Res 29:1433-1442

Lopes M, Foiani M, Sogo JM (2006) Multiple mechanisms control chromosome integrity after replication fork uncoupling and restart at irreparable UV lesions. Mol Cell 21:15-27

Lucas I, Feng W (2003) The essence of replication timing: determinants and significance. Cell Cycle 2:560-563

Marheineke K, Hyrien O (2001) Aphidicolin triggers a block to replication origin firing in xenopus egg extracts. J Biol Chem 276:17092-17100

Marheineke K, Hyrien O (2004) Control of replication origin density and firing time in Xenopus egg extracts: role of a caffeine-sensitive, ATR-dependent checkpoint. J Biol Chem 279:28071-28081
Maya-Mendoza A, Petermann E, Gillespie DA, Caldecott KW, Jackson DA (2007) Chk1 regulates the density of active replication origins during the vertebrate $\mathrm{S}$ phase. EMBO J 26:2719-2731

Michalet X, Ekong R, Fougerousse F, Rousseaux S, Schurra C, Hornigold N, van Slegtenhorst M, Wolfe J, Povey S, Beckmann JS, Bensimon A (1997) Dynamic molecular combing: stretching the whole human genome for highresolution studies. Science 277:1518-1523

Michel B, Boubakri H, Baharoglu Z, LeMasson M, Lestini R (2007) Recombination proteins and rescue of arrested replication forks. DNA Repair 6:967-980

Newlon CS, Theis JF (1993) The structure and function of yeast ARS elements. Curr Opin Genet Dev 3:752-758

Nieduszynski CA, Knox Y, Donaldson AD (2006) Genomewide identification of replication origins in yeast by comparative genomics. Genes Dev 20:1874-1879

Nieduszynski CA, S-i H, Ak P, Benham CJ, Donaldson AD (2007) OriDB: a DNA replication origin database. Nucleic Acids Res 35:D40-D46

Norio P, Schildkraut CL (2001) Visualization of DNA replication on individual epstein-barr virus episomes. Science 294:2361-2364

Norio P, Kosiyatrakul S, Yang Q, Guan Z, Brown NM, Thomas S, Riblet R, Schildkraut CL (2005) Progressive activation of DNA replication initiation in large domains of the immunoglobulin heavy chain locus during B cell development. Molecular Cell 20:575-587

Pasero P, Bensimon A, Schwob E (2002) Single-molecule analysis reveals clustering and epigenetic regulation of replication origins at the yeast rDNA locus. Genes Dev $16: 2479-2484$

Pillaire MJ, Betous R, Conti C, Czaplicki J, Pasero P, Bensimon A, Cazaux C, Hoffmann JS (2007) Upregulation of error-prone DNA polymerases beta and kappa slows down fork progression without activating the replication checkpoint. Cell Cycle 6:471-477

Raghuraman MK, Winzeler EA, Collingwood D, Hunt S, Wodicka L, Conway A, Lockhart DJ, Davis RW, Brewer BJ, Fangman WL (2001) Replication dynamics of the yeast genome. Science 294:115-121

Rao VA, Conti C, Guirouilh-Barbat J, Nakamura A, Miao Z-H, Davies SL, Sacca B, Hickson ID, Bensimon A, Pommier Y (2007) Endogenous \{gamma $\}-H 2 A X-A T M-C h k 2$ checkpoint activation in bloom's syndrome helicase deficient cells is related to DNA replication arrested forks. Mol Cancer Res 5:713-724

Semple JW, Da-Silva LF, Jervis EJ, Ah-Kee J, Al-Attar H, Kummer L, Heikkila JJ, Pasero P, Duncker BP (2006) An essential role for Orc6 in DNA replication through maintenance of pre-replicative complexes. EMBO J 25:5150-5158

Shechter D, Costanzo V, Gautier J (2004) ATR and ATM regulate the timing of DNA replication origin firing. Nat Cell Biol 6:648-655

Shimada K, Pasero P, Gasser SM (2002) ORC and the intra-Sphase checkpoint: a threshold regulates Rad53p activation in S phase. Genes Dev 16:3236-3252

Sidorova JM, Li N, Folch A, Monnat RJ Jr (2008) The RecQ helicase WRN is required for normal replication fork progression after DNA damage or replication fork arrest. Cell Cycle 7:796-807 
Tanaka S, Umemori T, Hirai K, Muramatsu S, Kamimura Y, Araki H (2006) CDK-dependent phosphorylation of Sld2 and Sld3 initiates DNA replication in budding yeast. Nature 445:328-323

Taylor JH (1977) Increase in DNA replication sites in cells held at the beginning of S phase. Chromosoma 62:291-300

Taylor JH, Miner P (1968) Units of DNA replication in mammalian chromosomes. Cancer Res 28:1810-1814

Tourriere H, Pasero P (2007) Maintenance of fork integrity at damaged DNA and natural pause sites. DNA Repair 6:900-913

Tourriere H, Versini G, Cordon-Preciado V, Alabert C, Pasero P (2005) Mrc1 and tofl promote replication fork progression and recovery independently of Rad53. Mol Cell 19:699706

Tuduri S, Crabbe L, Conti C, Tourriere H, Holtgreve-Grez H, Jauch A, Pantesco V, De Vos J, Theillet C, Pommier Y et al (2009) Topoisomerase 1 suppresses replication stress and genomic instability by preventing interference between replication and transcription. Nat Cell Biol 11:1315-1324
Versini G, Comet I, Wu M, Hoopes L, Schwob E, Pasero P (2003) The yeast Sgs1 helicase is differentially required for genomic and ribosomal DNA replication. EMBO J 22:1939-1949

Woodward AM, Gohler T, Luciani MG, Oehlmann M, Ge X, Gartner A, Jackson DA, Blow JJ (2006) Excess Mcm2-7 license dormant origins of replication that can be used under conditions of replicative stress. J Cell Biol 173:673683

Yabuki N, Terashima H, Kitada K (2002) Mapping of early firing origins on a replication profile of budding yeast. Genes Cells 7:781-789

Yamashita M, Hori Y, Shinomiya T, Obuse C, Tsurimoto T, Yoshikawa H, Shirahige K (1997) The efficiency and timing of initiation of replication of multiple replicons of Saccharomyces cerevisiae chromosome VI. Genes Cells 2:655-665

Zegerman P, Diffley JFX (2006) Phosphorylation of Sld2 and Sld3 by cyclin-dependent kinases promotes DNA replication in budding yeast. Nature 445:281-285 